\title{
Molecular dynamics simulation of the early stages of self-interstitial clustering in silicon
}

\author{
Luis A. Marqués ${ }^{1}$, María Aboy, Manuel Ruiz, Iván Santos, Pedro López, Lourdes Pelaz
}

Departamento de Electrónica, Universidad de Valladolid, E.T.S.I. de Telecomunicación, 47011 Valladolid, Spain

\begin{abstract}
We have studied the early stages of self-interstitial clustering in silicon using molecular dynamics simulation techniques. We have generated silicon samples of over 200000 atoms where we introduced a $0.5 \%$ extra concentration of self-interstitials. Then samples were annealed at several temperatures. During the simulations we observed the formation of interstitial clusters with different atomic structures, ranging from spherical and amorphous-like clusters, to highly ordered extended configurations such as (110) chains, $\{111\}$ rod-like defects and dislocation loops, and $\{100\}$ planar defects. This last type of defects, while common in germanium, have not been observed in silicon until very recently, in ultra-fast laser annealing experiments. The particular morphology of formed interstitial clusters is found to be related to the annealing temperature, as it is observed in the experiments. From the molecular dynamics simulations we have analyzed the atomic mechanisms leading to the formation and growth of interstitial clusters, with special attention to the newly found $\{100\}$ planar defects.
\end{abstract}

Keywords: Si; Annealing; Atomistic simulation; Molecular dynamics; Extended defects.

\section{Introduction}

Due to its technological relevance for the fabrication of Si devices, self-interstitial clustering in silicon after ion implantation has attracted much attention over the last decades [1-3]. It is well known that, apart from the dopant atoms, ion implantation injects self-interstitials in the lattice above equilibrium conditions. These excess interstitials form aggregates that evolve through an Ostwald ripening process: larger defect clusters grow at the expense of single interstitials freed from smaller and less stable agglomerates [4]. At sizes of several hundred interstitials these aggregates become visible in high-resolution transmission electron microscopy images, they show regular atomic structures, and are generally known as extended defects [5]. Extended defects are responsible for some deleterious effects such as the transient enhanced diffusion of dopants [6] and the presence of leakage currents in the final device [7].

The type of extended defects which can be observed in Si depends on the particular implantation and annealing conditions [5]. In general, at short anneals and low temperatures the most frequent are the so-called \{311\} defects, while for higher thermal budgets the dominant defects are $\{111\}$ dislocations. Recently, the use of ultra-fast laser annealing techniques has been proposed for the activation of ion implanted dopants (e.g. boron) in very thin layers near the silicon surface, without harming the underlying buried device layers (which is of critical importance for the fabrication of 3D integrated devices) [8]. Ultra-fast laser annealing introduce very high temperatures in localized regions of the substrate at the nanosecond time-scale. Under this extreme conditions, $\{100\}$ planar loops have been observed to form for the first time in pure Si, instead of the conventional $\{311\}$ and $\{111\}$ defects. These findings suggest that there is a relation between the annealing temperature and the type of extended defect formed. Most likely the formation of a particular extended defect is related to how self-interstitials cluster during the early stages of the aggregation process. Unfortunately, the small sizes and short time-scales involved preclude its direct study by experimental techniques. Precisely in these circumstances the use of simulation techniques can provide valuable information on the detailed atomistic mechanisms.

In this paper we have used the molecular dynamics (MD) simulation technique to get some fundamental

\footnotetext{
${ }^{1}$ Corresponding author. Tel.: +034-983185503; fax: +034-983423675. E-mail address: lmarques@ele.uva.es.
} 
understanding on the early stages of the self-interstitial aggregation process in Si. Details about the simulation setup are given in the next section. The main results of our study are presented in Section 3. We pay special attention to the formation and characterization of the newly found $\{100\}$ planar loops.

\section{Molecular dynamics simulations}

We started from a perfect Si lattice in a cell of size $158 \AA \times 161 \AA \times 161 \AA$. Axis orientations were X [100], Y [011] and Z [0-11]. We used periodic boundary conditions to minimize finite size effects. We introduced $0.5 \%$ of excess interstitial atoms in tetrahedral positions chosen at random. The final number of atoms in the simulation was 205627. Generated samples were annealed at different temperatures using the open-source MD software package LAMMPS [9], following the scheme of the work done by Kapur and Sinno [10]. These authors used the EDIP potential [11] in their MD simulations to describe Si-Si interactions, but they found that it was not able to predict the formation of $\{311\}$ defects at low temperatures, unlike it is observed in the experiments. We used instead in our MD simulations the Tersoff 3 potential [12]. It describes the strength of a given bond between two Si atoms as a function of the particular arrangement of other atoms around the bond. This allows correctly describing structures different from perfect crystal, as it is the case of intrinsic defects and the amorphous phase [13,14]. Unfortunately the Tersoff 3 potential overestimates the silicon melting point (2400 K instead of $1685 \mathrm{~K}$ ) [15]. However, this is not a serious drawback since it is possible to make a temperature re-scaling [16]:

$$
\mathrm{T}_{\text {Real }}(\mathrm{K})=\mathrm{T}_{\text {Tersoff }}(\mathrm{K}) \times 1685 / 2400
$$

Eq. 1

To identify and characterize defects in our simulations we used the scheme depicted in Fig. 1. First, we carry a time average of atom coordinates every 1000 time-steps, in order to eliminate thermal vibrations. Then we compare the averaged configuration with the perfect lattice. When an atom is closer than $0.7 \AA$ to a lattice site the atom is associated to that site, otherwise it is labeled as "displaced". In the same way, lattice sites with no associated atom are labeled as "empty". Finally, displaced atoms (DAs) and empty lattice sites are grouped within a first neighbor distance to form defects. The size of the defect is determined by the number of DAs, and the net number of excess interstitials by the difference between DAs and empty lattice sites.
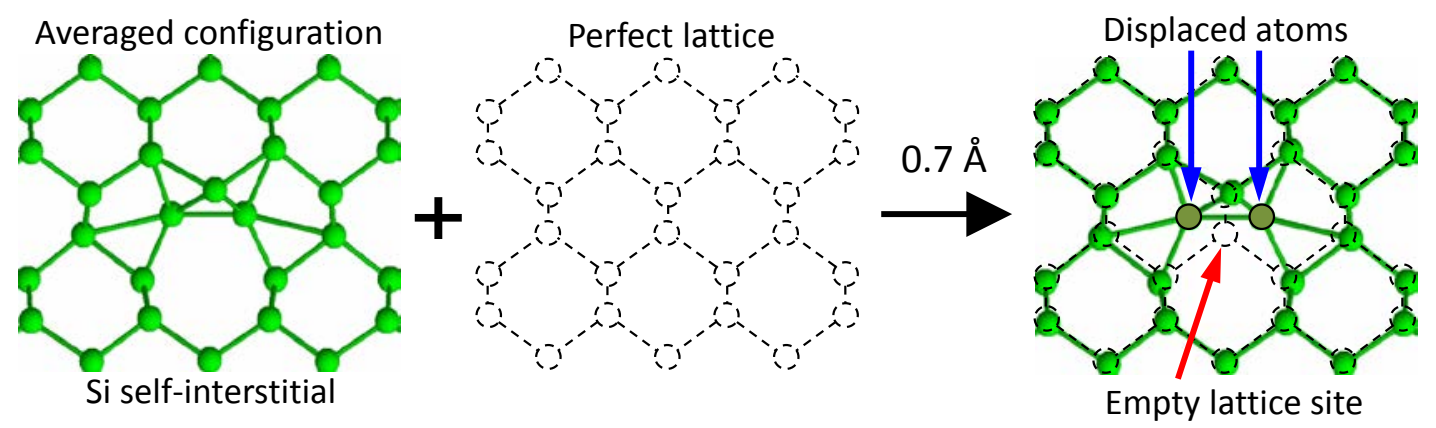

Fig. 1. Scheme used for defect identification and characterization, illustrated for the particular case of a Si dumbbell interstitial.

\section{Results and discussion}

Figure 2 shows several snapshots corresponding to the annealing of the initial sample at a low temperature, $1200 \mathrm{~K}$ (equivalent to a real temperature of around $800 \mathrm{~K}$ ). For the sake of clarity only DAs are shown. Isolated Si self-interstitials and early formed di-interstitials are highly mobile species, so they diffuse and interact giving rise to larger defects. The aggregation process continues until all mobile species are captured by larger and immobile clusters. After 170 ns of annealing, a total of 150 different defects are formed with a relatively small interstitial content (most defects have less than 15 interstitials). Larger clusters are $\{111\}$ rod-like defects (RLDs), the precursors of $\{111\}$ dislocation loops [5]. A lot of (110) chains are also observed, which are the precursors of \{311\} defects [2]. But most interstitials are in the form of tetra-interstitials in the Arai configuration [17], which is a very 
stable defect cluster where all atoms are four-fold coordinated (see Fig. 3). There are also small agglomerates of tetra-interstitials. From here, cluster growth will proceed by an Ostwald ripening process [4]: stable defects will capture interstitial emitted by less stable clusters. In this way some defects will grow in size at the expense of others that will shrink. Unfortunately, the full ripening process cannot be simulated using MD techniques since involved time-scales are too large.

(a) $t=100 p s$

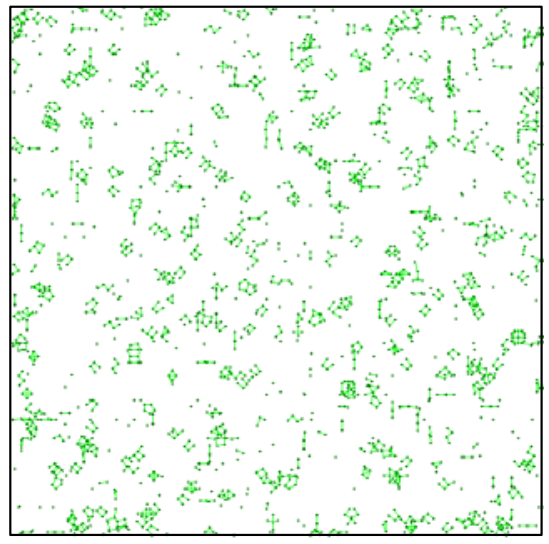

(b) $\mathrm{t}=10 \mathrm{~ns}$

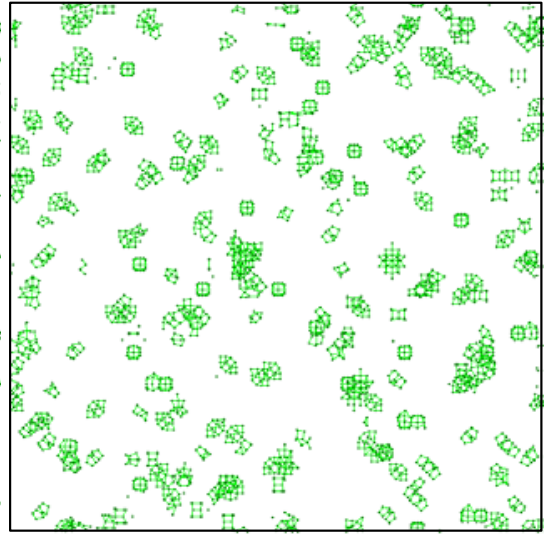

(c) $\mathrm{t}=170 \mathrm{~ns}$

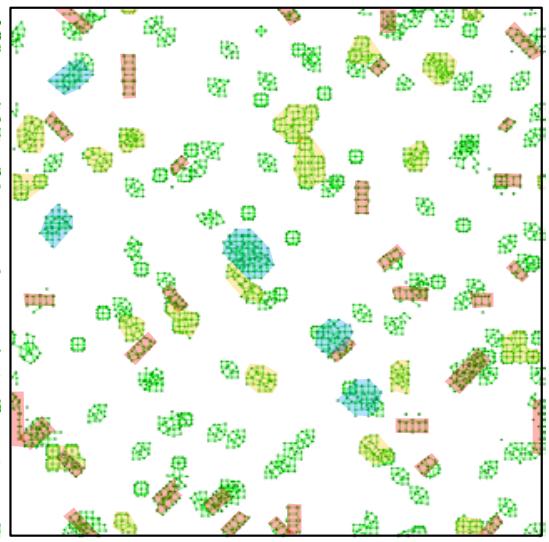

Fig. 2. ZY projections of the DAs within the simulation cell during the annealing at $1200 \mathrm{~K}$ at three different times. In snapshot (c), defects shadowed in blue are $\{111\}$ RLDs, in red are (110) chains, and in yellow small agglomerates of tetra-interstitials. Remaining defects are isolated tetra-interstitials.

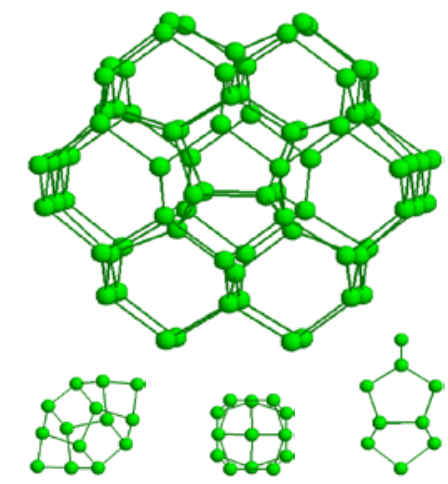

Fig. 3. Atomic configuration of the Arai tetra-interstitial in Si. At the bottom , different views of this defect when displaying only DAs. First two images are projections on the $<100>$ direction, and last one on the $<110>$ direction.

In the case of annealing at high temperatures, the interstitial clustering process is completely different. Figure 4 shows several snapshots corresponding to the annealing of the initial sample at $1900 \mathrm{~K}$ (equivalent to a real temperature of around $1300 \mathrm{~K}$ ). Again only DAs are shown. After 3 ns of annealing, apart from the very stable tetrainterstitials, big amorphous clusters are formed. These are highly mobile, sweeping smaller interstitial clusters in their way. Some (110) chains are formed, but at this high temperature they are not stable enough to survive. At the end of the clustering process at 30 ns fewer but much larger interstitial defects are generated. Almost half of the initial interstitials end up in $\{111\}$ clusters of two types: RLDs and Frank loops. The other half end up forming large $\{100\}$ loops, not observed at low temperatures. It is worth to note that all these defects produce a strong strain field around them: atoms above and below the defect planes are in perfect tetrahedral positions, but they are shown as DAs because they have been pulled by the defect to distances larger than $0.7 \AA$ with respect to their original crystal lattice positions. 
(a) $\mathrm{t}=100 \mathrm{ps}$

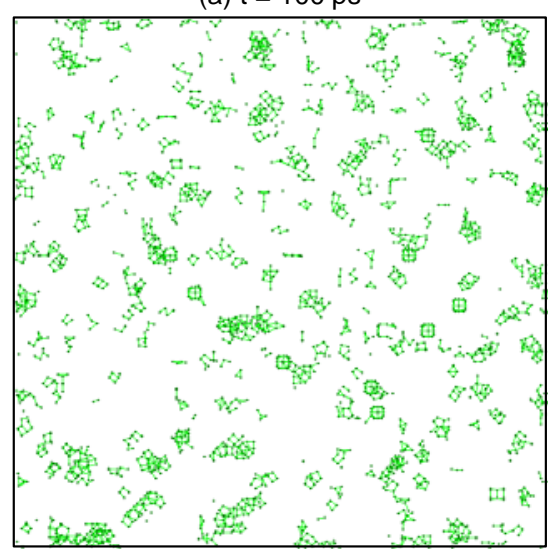

(b) $t=3 n s$

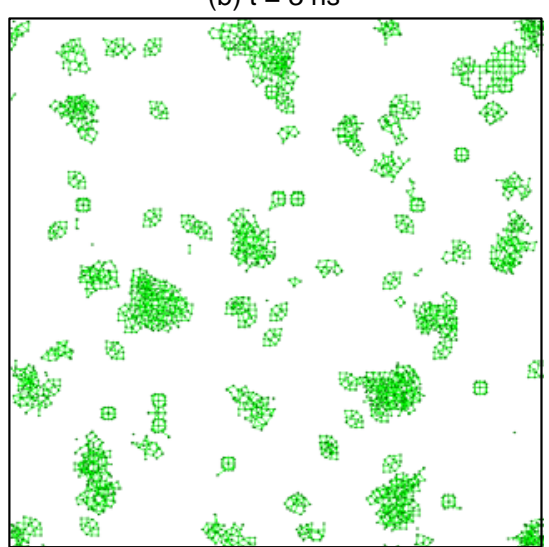

(c) $\mathrm{t}=30 \mathrm{~ns}$

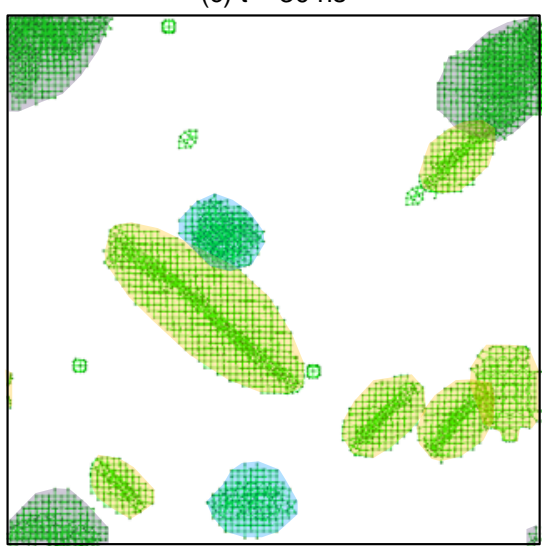

Fig. 4. ZY projections of the DAs within the simulation cell during the annealing at $1900 \mathrm{~K}$ at three different times. In snapshot (c), defects shadowed in blue are $\{111\}$ RLDs, in purple are $\{111\}$ Frank loops, and in yellow $\{100\}$ loops. Remaining defects are isolated tetra-interstitials.

From our MD simulations at high temperature, we analyze in more detail how $\{100\}$ loops are generated. Figure 5 shows several snapshots taken during annealing at $1900 \mathrm{~K}$ depicting the formation of a $\{100\}$ loop. During the early stages of the interstitial aggregation process, highly mobile amorphous interstitial clusters are formed, such as the one illustrated in Fig. 5(a). Once in a while some tetra-interstitials nucleate in the defect edges. Frequently these nucleated tetra-interstitials dissolve again into the amorphous structure. But sometimes they survive long enough to promote the amorphous cluster crystallization. For the case of the cluster shown in Fig. 5, after the nucleation of two tetra-interstitials, the whole defect undergoes a phase transformation from an amorphous to a fully ordered structure in just a few picoseconds. This process is similar to the explosive crystallization of an amorphous material [18], where first a crystal seed of critical size has to be nucleated. Then, the seed starts growing leading to the fast crystallization of the surrounding amorphous phase.

(a) $\mathrm{t}=12.87 \mathrm{~ns}$
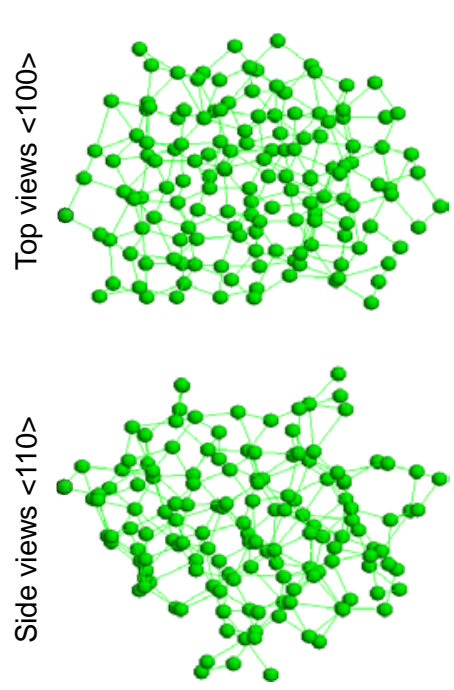

(b) $t=12.94 n s$
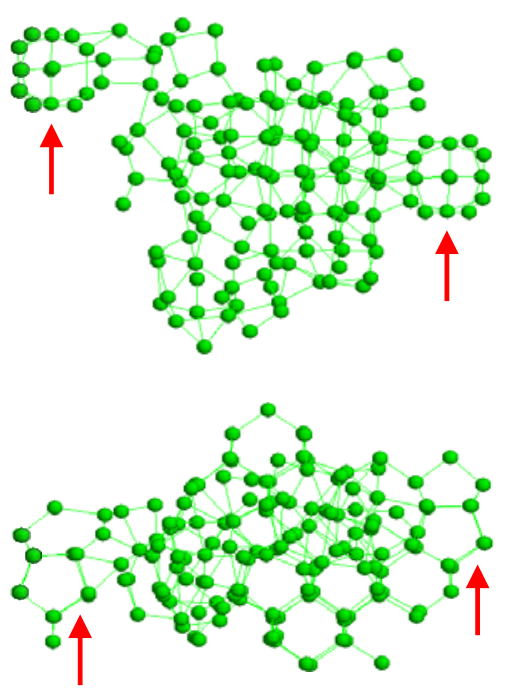

(c) $\mathrm{t}=14.11 \mathrm{~ns}$

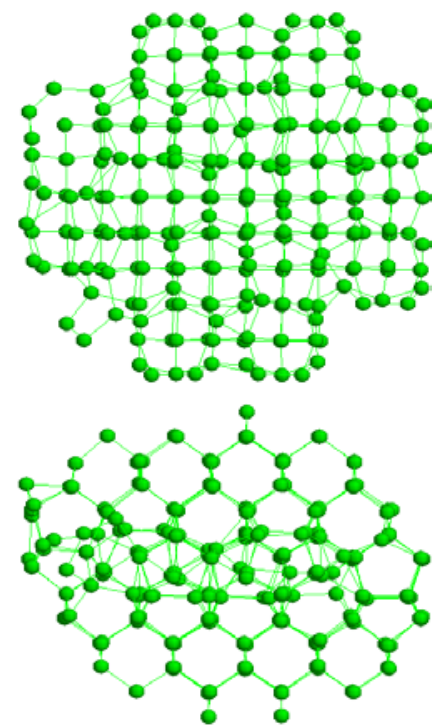

Fig. 5. Snapshots showing the formation of a $\{100\}$ loop during annealing at $1900 \mathrm{~K}$. Only DAs are shown. (a) Initial amorphous interstitial cluster, (b) two tetra-interstitials nucleate in the amorphous cluster edges (indicated by the red arrows), and (c) the final and fully ordered structure of the $\{100\}$ loop. 
Figure 5(c) shows both the top and side views of a typical $\{100\}$ loop formed during the annealing at $1900 \mathrm{~K}$. In principle, the loop appears to be an agglomeration of Arai tetra-interstitials located in alternate positions within the crystal lattice, like in a chess board. Similar atomic arrangements are observed for the rest of the $\{100\}$ loops formed in the MD simulation. However, a closer look indicates that the typical structure of the Arai tetra-interstitial is only maintained in the loop edges. In the loop core, thermal agitation favors the reordering of atoms from the tetra-interstitial structure to the atomic arrangement shown in Fig. 6. From our simulations we have measured a Burgers vector of one third the lattice unit $a$ in the [001] direction, in fair agreement with the experimental measurement of $\sim a / 4$ [001] by Qiu and coworkers [8].

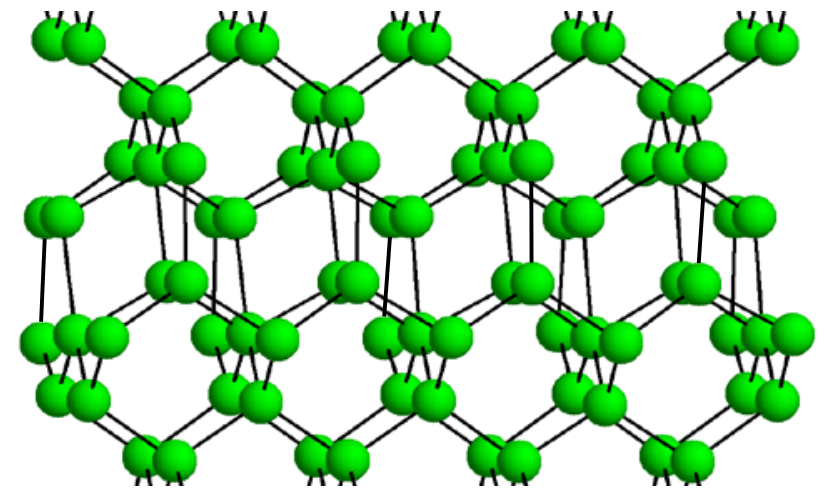

Fig. 6. Atomic structure of the $\{100\}$ loops.

\section{Conclusions}

We have used MD simulation techniques to study the early stages of self-interstitial aggregation in Si. Annealing at low temperatures produces many interstitial clusters of small size. Formed defects are (110) chains (precursors of the $\{311\}$ defects), $\{111\}$ RLDs (precursors of $\{111\}$ dislocations), and small agglomerates of tetra-interstitials. The growth mechanism at low temperature is mediated by the diffusion of very small mobile clusters, mostly single selfinterstitials and di-interstitials. In turn, annealing at high temperatures produces fewer and much larger clusters: \{111\} RLDs and Frank loops, and $\{100\}$ loops. No $\{311\}$ defects are observed, in agreement with the experimental observations. In particular, the generation of $\{100\}$ loops is caused by the explosive crystallization of amorphous clusters at high temperatures, initiated by the spontaneous nucleation of tetra-interstitials at the clusters edges. From the analysis of the simulations we have obtained the atomic arrangement within the $\{100\}$ loops.

\section{Acknowledgement}

This work has been funded by the Spanish Ministerio de Ciencia e Innovación under Project No. TEC201127701, and the Junta de Castilla y León under Project No. VA331U14.

\section{References}

1. K. S. Jones, S. Prussin, and E. R. Weber, Appl. Phys. A 45, 1 (1988).

2. S. Takeda, Micros. Res. Techniq. 40, 313 (1998).

3. The International Technology Roadmap for Semiconductors, http://www.itrs.net/.

4. N. E. B. Cowern, G. Mannino, P. A. Stolk, F. Roozeboom, H. G. A. Huizing, J. G. M. van Berkum, F. Cristiano, A. Claverie, and M. Jaraiz, Phys. Rev. Lett. 82, 4460 (1999).

5. A. Claverie, B. Colombeau, B. D. Mauduit, C. Bonafos, X. Hebras, G. B. Assayag, and F. Cristiano, Appl. Phys. A 76, 1025 (2003).

6. D. J. Eaglesham, P. A. Stolk, H.-J. Gossmann, and J. M. Poate, Appl. Phys. Lett. 65, 2305 (1994).

7. C. Nyamhere, F. Cristiano, F. Olive, E. Bedel-Pereira, J. Boucher, Z. Essa, D. Bolze, Y. Yamamoto, AIP Conf. Proc. 1496, 171 (2012). 
8. Y. Qiu, F. Cristiano, K. Huet, F. Mazzamuto, G. Fisicaro, A. La Magna, M. Quillec, N. Cherkashin, H. Wang, S. Duguay, and D. C. Blavette, Nano Lett. 14, 1762 (2014).

9. S. Plimpton, J. Comp. Phys. 117, 1 (1995).

10. S. Kapur and T. Sinno, Phys. Rev. B 82, 045205 (2010).

11. M. Z. Bazant, E. Kaxiras, and J. F. Justo, Phys. Rev. B 56, 8542 (1997).

12. J. Tersoff, Phys. Rev. B 38, 9902 (1988).

13. L. A. Marqués, L. Pelaz, M. Aboy, L. Enríquez, and J. Barbolla, Phys. Rev. Lett. 91, 135504 (2003).

14. K. J. Dudeck, L. A. Marqués, A. P. Knights, R. M. Gwilliam, and G. A. Botton, Phys. Rev. Lett. 110, 166102 (2013).

15. L. A. Marqués, L. Pelaz, M. Aboy, and J. Barbolla, Nucl. Instrum. Methods Phys. Res. B 216, 57 (2004).

16. L. J. Porter, S. Yip, M. Yamaguchi, H. Kaburaki, and M. Tang, J. Appl. Phys. 81, 96 (1997).

17. N. Arai, S. Takeda, and M. Kohyama, Phys. Rev. Lett. 78, 4265 (1997).

18. W. C. Sinke, A. Polman, S. Roorda, and P. A. Stolk, Appl. Surf. Sci. 43, 128 (1989). 